\title{
Sparse Network Equivalent Based on Time-Domain Fitting
}

\author{
Wallace do Couto Boaventura, Member, IEEE, Adam Semlyen, Life Fellow, IEEE, \\ M. Reza Iravani, Senior Member, IEEE, and Amauri Lopes, Member, IEEE
}

\begin{abstract}
The paper presents an approach for network equivalent calculation for the analysis of electromagnetic transients in power systems. It is based on time-domain fitting and enforces some degree of sparsity while preserving the accuracy of the equivalent. The calculated equivalent is appropriate for direct interface with the rest of the system in time-domain. A constrained least squares solution of the time-domain fitting equations is used to ensure accuracy at 0 and $60 \mathrm{~Hz}$. Results demonstrating the accuracy and computational efficiency of the method are presented.
\end{abstract}

Index Terms-Constrained least squares, discrete time, electromagnetic transients, network equivalent, time-domain fitting.

\section{INTRODUCTION}

$\mathbf{N}$ ETWORK equivalents are used to facilitate the computation of electromagnetic transients in power systems. They can be obtained using either frequency-domain [1], [2] or timedomain [3]-[8] fitting techniques. Various methods relevant to network equivalent calculations with their merits and limitations are described in [1]-[8].

This paper presents a new formulation for deriving network equivalents based on time-domain fitting procedures. The required data is obtained either from computed or measured time-domain response of the network. The presented method enforces some degree of sparsity while preserving the accuracy of the equivalent. To allow interfacing between the equivalent and the remainder of the system, for the purpose of digital time-domain simulation of electromagnetic transients, the equivalent is derived in discrete-time domain. The main feature of the new equivalent is its computational efficiency especially when a small time step is needed. Several issues regarding its calculation, like the order, stability, passivity, sparsity, and accuracy of the equivalent, are discussed and specifically the problems of sparsity and accuracy are carefully examined. Computational efficiency and accuracy in the context of electromagnetic transients analysis have been discussed in [9]-[15].

Manuscript received December 7, 1999. This work was supported by the Natural Sciences and Engineering Research Council of Canada and CAPES, Brazil.

W. do Couto Boaventura is with the Department of Electrical and Computer Engineering, the University of Toronto, Toronto, ON M5S 3G4, Canada (e-mail: boavenw@power.ele.utoronto.ca) on leave from the Department of Electrical Engineering, Federal University of Minas Gerais, 31.270-010 Belo Horizonte, MG, Brazil.

A. Semlyen and M. R. Iravani are with the Department of Electrical and Computer Engineering, University of Toronto, Toronto, ON M5S 3G4, Canada (e-mail: semlyen@ecf.utoronto.ca; iravani@ecf.utoronto.ca).

A. Lopes is with the Department of Communications, University of Campinas, 13.083-970 Campinas, SP, Brazil (e-mail: amauri@decom.fee.unicamp.br).

Publisher Item Identifier S 0885-8977(02)00586-1.
The rest of this paper is organized as follows. Section II presents the details of the equivalent calculation. In Section III, results are given for validating the methodology in terms of accuracy and efficiency as well as to show its limitations. Conclusions are stated in Section IV.

\section{NeTwork EQuivalent CALCUlation}

The equivalent considered in this paper is for linear networks. A linear system, seen as a single port network, can be fully characterized in the discrete-time domain by a linear constant-coefficients difference equation, if time invariance and zero initial state are assumed [16]. Discrete-time domain is used here since it is closely related to the kind of data assumed for the equivalent. If the data is obtained from a transient calculation program, using a fixed time step, it is considered as accurate information for the system under study, disregarding the errors incurred by the discretization. When the data comes from measurement, the errors due to digitization and noise play a more important role than that of the discretization. Considering that two data sequences of length $N$ are available, a voltage/current pair $v(n)$ and $i(n)$ taken at the port of the external subsystem, the following difference equation of order $p$ (related to the output) characterizes the external subsystem [16] and the equivalent:

$$
\sum_{k=0}^{p} a_{k} i(n-k)=\sum_{k=0}^{q} b_{k} v(n-k) \quad(n=1, \ldots, N)
$$

where $q$ is the number of past terms in the input and $a_{k}$ and $b_{k}$ are the coefficients that describe the equivalent and to be determined. The external and study subsystems identify portions of a system to be equivalenced and to be represented in full, respectively. Rearranging the terms in (1), setting the value of $a_{0}$ to one , and $q=p$ (see Section II-D below), we get the normalized equation

$$
i(n)=b_{0} v(n)+\sum_{k=1}^{p}\left(b_{k} v(n-k)-a_{k} i(n-k)\right) .
$$

A unit step voltage is used to produce the above sequences because it can be easily represented in transient calculation programs or obtained by pulse generators when measurements are used. Moreover, it permits to obtain the characteristics of the system in the desired frequency range. The coefficient $b_{0}$ in (2) has the dimension of admittance and the term under the summation is calculated only from past values. Equation (2) is in the appropriate form to integrate the equivalent into a transient calculation program. The calculation of the equivalent boils down 
to identifying $a_{k}$ and $b_{k}$. In the following, a procedure to determine $a_{k}$ and $b_{k}$, while ensuring the sparsity and accuracy of the equivalent, is presented. For multiphase systems, equivalents can be obtained using this procedure by treating each mode separately via modal decomposition.

\section{A. Identification of $a_{k}, b_{k}$}

Using (1), we can build a set of $N$ linear equations, for each value of $n$ from zero to $N-1$. This leads to (3), as shown at the bottom of the page, where the subscripts in the variables $v$ and $i$ indicate the time index in the sequences or

$$
\left[\begin{array}{ll}
C & -V
\end{array}\right]\left[\begin{array}{l}
a \\
b
\end{array}\right]=[-i]
$$

This method of solution is related to Prony's work [17], which deals with the impulse response of linear systems and would lead to a different set of equations. In Prony's method, the upper part of the submatrix $V$ (first $p+1$ lines) from (3) is reduced to the identity matrix and the coefficients $b_{k}$ are only a linear combination of the coefficients $a_{k}$. Usually, $N$ is greater than $2 p+1$. Thus, (3) is overdetermined and a solution in the least squares sense can be obtained for the unknowns $a_{k}$ and $b_{k}$. Our studies indicate that such solution results in a good overall accuracy even for $p$ as large as 250 and $N=1000$. Then, the number of columns of the matrix from (3) is 501, according to the 501 unknowns $\left(a_{k}, b_{k}\right)$, and gives a redundancy close to two. Obtaining a solution for $a_{k}$ and $b_{k}$, one should evaluate if: 1) the desired accuracy is achieved, including the system admittance values at zero and power frequencies, and 2) the deduced equivalent is both stable and passive.

The length $N$ of the data vectors $v(n)$ and $i(n)$ is not limited regarding their generation. It is however time and memory consuming to solve overdetermined systems larger than those obtained for $p=250$ and $N=1000$. We have found this as a suitable limit in view of the computational power of current personal computers. If $N=1000$, the total computation/measurement time will then be determined by the time step $\Delta t$. It is tied to the smallest time constant or travel time of the system. These quantities, $N$ and $\Delta t$, determine if the vectors $v(n)$ and $i(n)$ have a frequency content suitable to obtain a network equivalent that accurately represents the external subsystem admittance at single frequencies like zero and power frequency $(60 \mathrm{~Hz})$. For instance, if a $10 \mu$ s time step is used, the total time will be $10 \mathrm{~ms}$, which can characterize the system response at $60 \mathrm{~Hz}$, but lacks the information concerning the correct value for the zero frequency. As this is usually the case, a constrained solution of the overdetermined system (3) can be used to ensure the accuracy at both zero and $60 \mathrm{~Hz}$ frequencies. The details of this procedure are as follows.

\section{B. Single Frequency Constraint for the Identification of $a_{k}, b_{k}$}

If the complex value $Y_{0}$ of the external subsystem admittance is accurately known for a single frequency $\omega_{0}$, it can be used as a constraint for solving (3). This ensures the accuracy of the equivalent at $\omega_{0}$. The constraint equations are derived from the analysis of the external subsystem excited by a single frequency voltage source

$$
v(n)=v\left(t_{n}\right)=v(n \Delta t)=v_{0} e^{j \omega n \Delta t}=v_{0}\left(e^{j \omega \Delta t}\right)^{n} .
$$

Using the auxiliary variable $z=e^{j \omega \Delta t}$, we have

$$
v(n)=v_{0} z^{n}
$$

Similarly, the corresponding current can be expressed as

$$
i(n)=i_{0} z^{n}
$$

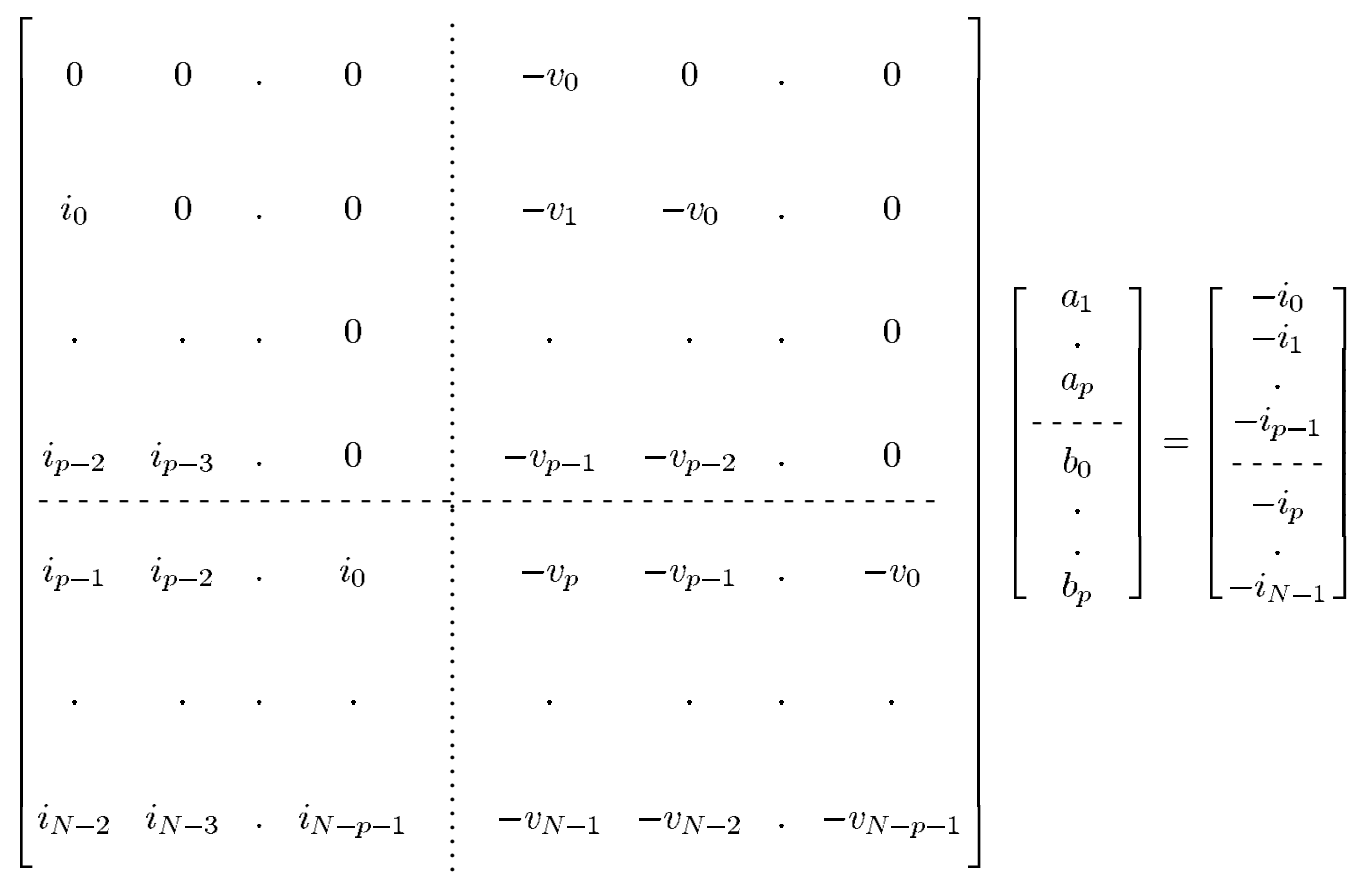


Setting $\omega=\omega_{0}, v_{0}=1$, which implies $i_{0}=Y_{0}$, and substituting (6) and (7) in (2), we obtain

$$
Y_{0}=b_{0}+\sum_{k=1}^{p}\left(b_{k}-Y_{0} a_{k}\right) z^{-k} .
$$

Splitting (8) in its real and imaginary parts results in two equations in terms of unknowns $a_{k}, b_{k}$ to be used as equality constraints for solving (3). In this paper, (8) is used to enforce the accuracy of the equivalent for the external subsystem admittance at zero [then (8) is real] and power frequencies. Thus, the complete formulation for calculating $a_{k}$ and $b_{k}$ as a constrained least squares solution of an overdetermined set of linear equations is derived. In general, this problem can be written as

$$
\min _{x}\|A x-b\|, \quad D x=e
$$

where $D$ and $e$ are obtained from the constraint equations using (8). A solution for (9) can be obtained using software packages, e.g., the Matlab [18] optimization toolbox. If a tolerance $\pm \mu$ is desired for the equality constraints in (9), it can be implemented using two inequalities, $D x<e+\mu$ and $D x>e-\mu$.

\section{Sparsity in the Identification of $a_{k}, b_{k}$.}

The described calculation method usually leads to an accurate network equivalent with respect to the overall fitting and the admittance values for zero and power frequencies. When used in the simulation of transients, the equivalent requires less computational effort than the calculations with the total system would require. However, if the ultimate goal is speed rather than maximal accuracy, a sparse network equivalent can be obtained. This requires a few modifications of the above procedure. There is a tradeoff between the degree of sparsity and accuracy: the higher the sparsity, the lower the accuracy. The constrained least squares procedure restricts the lack of accuracy to the overall fitting only, permitting high accuracy on the zero and power frequencies admittance values. The sparse network equivalent usually has the same or higher dynamic order $p$ as the nonsparse equivalent, but at a reduced computational burden.

The procedure to calculate a sparse equivalent is to eliminate some of the unknowns $a_{k}$ and $b_{k}$ by setting them to zero, as follows.

Step 1) Obtain a basic solution for $a_{k}$ and $b_{k}$ using (3), but no equality constraints.

Step 2) For the desired sparsity $N_{S}$ (number of nonzero coefficients for each set $a_{k}$ and $b_{k}$ ), $a_{k}$ and $b_{k}$ calculated from Step 1 are scanned to identify the positions of the $N_{S}$ largest coefficients. These values are of course not the best ones and they have to be recalculated as follows.

Step 3) Modify matrices $C, V$ from (4), and $D$ from (9), keeping only the columns corresponding to the $N_{S}$ largest coefficients for $a_{k}$ and $b_{k}$.

Step 4) Solve (9), which includes the constraints to achieve the desired accuracy for the admittance values at zero and power frequencies. The remaining coefficients are set to zero, resulting in sparse sets of coefficients $a_{k}$ and $b_{k}$.
Step 5) Check for stability and passivity of the equivalent, using the criteria presented in Section II-E. Check for the desired overall fitting error. If the stability, passivity, and fitting requirements are not satisfied, $N_{S}$ is increased and the procedure is repeated from Step 2 to Step 5 iteratively until the requirements are satisfied.

Equation (3) does not usually result in a full rank problem, especially when the ground mode is considered (see Section II-D below). Then the basic solution from Step 1 is best obtained using methods based on QR decomposition. This is due to the fact that these methods can automatically set to zero a number of variables corresponding to the rank deficiency [18], thus, providing $a b$ initio some sparsity.

\section{Determination of the Order of the Equivalent}

The order of the equivalent $p$ from the difference equation (1) determines the number of variables to be calculated and the rank condition of (4). The number of variables to be calculated is equal to $2 p+1$. As shown before, we use the same order for both sets of coefficients $a_{k}$ and $b_{k}$. This is not a restrictive assumption; it is based on the analysis of the current wave reflections in an open-ended single-phase transmission line excited by an ideal voltage source. Thus, to account for time delays and multiple reflections due to the transmission line, one must use the same order for $a_{k}$ and $b_{k}$. This analysis also provides information about the order of the equivalent. Hence, the order must be twice the travel time of the transmission line divided by the time step. It can be observed from this simple example that the order of the equivalent is determined by the occurrence of time related events such as arrival of the reflected current wave at the sending bus. As the velocity of propagation of the line mode is larger than that of the ground mode, one expects larger order for the latter, since the reflected waves take longer to arrive at the sending bus. A larger order implies more variables to be determined and the rank of (4) should increase accordingly. However, the analysis of the condition of the matrices in (4) for the line and ground modes indicates a lower rank for the ground mode. This is because the corresponding high loss attenuates the traveling waves and degrades the information associated with farther nodes. In the following, this problem is further examined and two approaches for determination of $p$ are discussed.

One approach to determine a suitable range for the order of $a_{k}$ and $b_{k}$ utilizes the "length" in time steps of the network and the number of lumped energy storage components. The lower limit for the "length" of a network is defined as the double of the time required for a signal to arrive from the boundary bus to the farthest node in the network through the shortest path possible, divided by the time step. This approach is adequate for radial networks only. If there are loops in the network, then the order could be close to the total length of the network. This is equal to the double of the sum of all transmission line travel times divided by the time step. This is an upper limit. Usually, a suitable value for the order is between these two limits.

The second approach is based on the rank analysis of the matrices of (4) via singular value decomposition (SVD) [19]. The data from $i(n)$ or $v(n)$, used to build $C$ or $V$, is corrupted by roundoff noise due to the limited number of significant digits 
(usually six) available in the output format from transient calculation programs. The effect of noise is easily noticed using SVD analysis and a procedure to determine $p$ based on this information is formulated in the following. Some remarks are important to be made with respect to the use of SVD.

1) The upper partitions of $C$ and $V, C_{U}$ and $V_{U}$, contribute to the rank of (4) with $p$ linearly independent rows, regardless of how large $p$ is, due to the upper triangles filled with zeros. These zero values account for the initial conditions. If (4) is well conditioned, the remaining $N-p$ lines should have other $p+1$ linearly independent rows, completing the rank. Therefore, the information about the rank must be searched in the lower partitions $\left(C_{L}\right.$ and $\left.V_{L}\right)$ of matrices $C$ and $V$. The upper partitions should not be used for obtaining $p$ because of their triangular shape. If $p$ is overestimated, they lead to erroneous information regarding the intrinsical order of the system.

2) The SVD analysis can be performed on the lower partitions $C_{L}$ and $V_{L}$, either separately or together. It reveals reliably the rank of the partition being used. If the source used to obtain $i(n)$ and $v(n)$ is a voltage source, one uses the data from $i(n)$-matrix $C_{L}$. In the case of a current source, one uses the data from $v(n)$-matrix $V_{L}$. If a complex source is used, the SVD analysis must be performed on the whole lower partition (matrices $C_{L}$ and $V_{L}$ ).

3) The order determined by SVD reflects the network topology and "length." If frequency dependent (FD) models are used, the SVD analysis also reflects the presence of losses and lumped elements in the FD model formulation. Since losses for the line mode are not very high, the SVD indicates a reliable value for $p$. However, the value of $p$ for the ground mode indicated by the SVD is not reliable. Despite the increased travel times, due to the high losses, the SVD leads to a $p$ smaller than that of the line mode. Therefore, the SVD analysis should not be used to determine $p$ for the ground mode. Instead, a value greater than that obtained for the line mode should be used. The choice of this value is made taking into account the information provided by the differences in the travel times. Determination of $a_{k}$ and $b_{k}$ for the ground mode is usually a rank deficient problem.

In the following, a procedure to determine $p$ for the line mode is presented using partition $C_{L}$.

Step 1) Build $C_{L}$ for a $p$ much larger than the order assumed for the external subsystem, for instance, equal to half the number of available data points, which is presumably much higher than the correct order for the system.

Step 2) Calculate the SVD of $C_{L}$.

Step 3) Identify the singular value which is about $10^{4}$ times larger than the smallest singular values (corresponding to the roundoff noise). Then, $p+1$ is equal to the index of this singular value.

Usually, the larger the order, up to a certain limit, the higher the accuracy. A plot of the singular values of partition $C_{L}$ (unless otherwise stated, built for $p=400$ and $20 \mu$ s time step) corresponding to the Case I of Section III is shown in Fig. 1. To

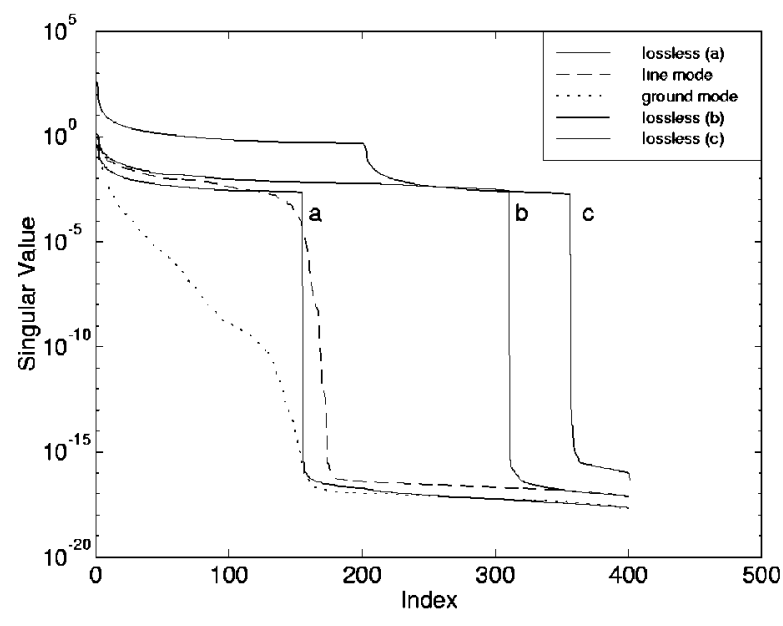

Fig. 1. SVD for the four transmission-line network of Case I in Section III.

show the influence of the time step in the order of the equivalent, $C_{L}$ is built from simulation using lossless models and two different time steps, 20 and $10 \mu \mathrm{s}$, respectively, for curves "lossless (a)" and "lossless (b)." The singular values for the line and ground modes (FD models) are also shown in Fig. 1. When the complete matrices $C$ and $V$ are used, the index of the singular value identified in Step 3 above is equal to $2 p+1$. The curve "lossless (c)" shows the singular values for this case (built for $p=200$ ). The influence of: 1) the time step; 2) the losses in the ground mode; and 3) overestimating $p$ with respect to the of the order of the equivalent is clearly shown in Fig. 1. In the case of overestimated $p$, the curve "lossless (c)" leads to 178 for the order, while the correct value, 155 , is indicated by the curve "lossless (a)."

We conclude by emphasizing that the order $p$ we choose for the realization (2) of an external subsystem must not be smaller than its intrinsical order. In the computational problem (3) or (4), because both $a_{k}$ and $b_{k}$ appear as unknowns, we have matrices with $2 p+1$ columns. Their numerical rank is increased accordingly due to their upper part (as discussed before) and only their lower part properly reflects the intrinsical rank or order of the network.

\section{E. Stability and Passivity Requirements}

Stability and passivity are inherent characteristics of a passive network and an equivalent for such a network must exhibit these characteristics. The stability of a linear system described by a constant-coefficients difference equation can be checked by the analysis of its natural modes corresponding to its zero-input response. The zero-input response of a system described by (1) is a sequence $i_{h}(n)$ that satisfies

$$
\sum_{k=0}^{p} a_{k} i_{h}(n-k)=0 .
$$

As in the case of constant-coefficients differential equations, (10) is referred to as the homogeneous equation and $i_{h}(n)$ is the homogeneous solution [16]. We seek a solution for (10) of the form

$$
i_{h}(n)=\text { const. } z^{n} \text {. }
$$


Substituting (11) in (10) results in the requirement that the complex numbers $z$ be the roots of the polynomial equation

$$
\sum_{k=0}^{p} a_{k} z^{-k}=0
$$

For stable sequences, the absolute value for each $z$ must be less than one. Similarly, if the equivalent represents an impedance instead of an admittance, the input is the current while the output is the voltage and the equation regarding stability is

$$
\sum_{k=0}^{p} b_{k} z^{-k}=0
$$

The requirement for passivity can be derived from (8) by rewriting it for any frequency $\omega$ and rearranging the terms. This leads to

$$
Y_{\omega}=G_{\omega}+j B_{\omega}=\frac{\sum_{k=0}^{p} b_{k} z^{-k}}{1+\sum_{k=1}^{p} a_{k} z^{-k}} .
$$

$G_{\omega}$ is the real part of the polynomial fraction in (14). The criterion for passivity of the equivalent is $G_{\omega}>0$. This must be checked for a reasonable number of frequencies (typically 1000) in the range from zero to $\pi / \Delta t$.

The procedure presented in this paper does not address the issues of stability and passivity as constraints imbedded in the calculation of the equivalent. However, a stable and passive equivalent is usually obtained if the following basic guidelines are observed.

1) Losses must be represented in the model adopted for the network under consideration.

2) If 1000 values for $i(n), v(n)$ are used, the upper limit of the length of the network, as previously defined, should not exceed 300. This allows a redundancy around two for the least squares solution in (9).

The size of (4), when the length of the network is larger than 300 , increases accordingly if the same degree of redundancy is used. For instance, if the length of the network is 600 , the resulting size of the combined matrices $C$ and $V$ is $2000 \times 1201$, since 2000 data points for $v(n)$ and $i(n)$ are used. Problems of this size are difficult to solve by an ordinary desktop computer.

\section{ILLUSTRATIVE EXAMPLES}

Two study cases, Cases I and II, are presented in this section to demonstrate the accuracy, speed, and limitations of the equivalencing technique. Fig. 2 shows a single-line diagram of a three-phase transmission system used for both study cases. The system is divided into a study subsystem (Line 1) and an external subsystem (rest of the lines). The objective of the study cases is to calculate equivalents for the external subsystem. All the transmission lines are assumed to be balanced.

To verify the accuracy and computational efficiency of the equivalent, a detailed time-domain electromagnetic transients simulation model of the test system is also developed and the corresponding results are compared. FD models [9], [10] are

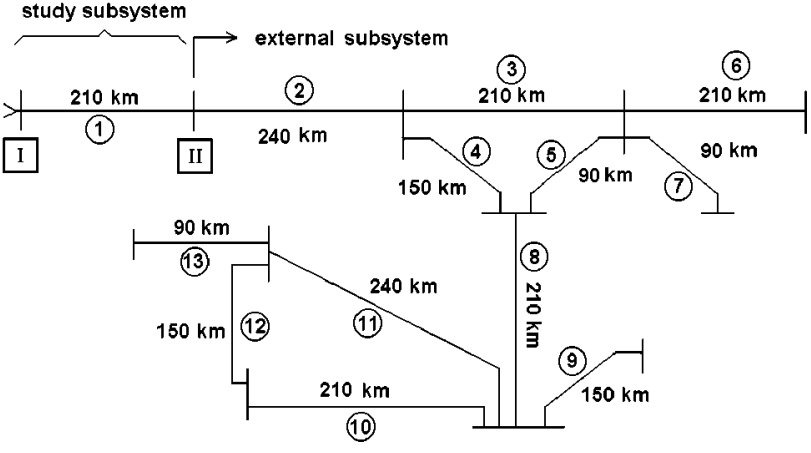

Fig. 2. Single-line diagram of the three-phase study system.

used for all lines and the propagation functions and characteristic impedances are represented by ten partial fractions for both line and ground modes. The partial fractions are obtained using vector fitting [20]. The routines for: 1) obtaining the network equivalent and 2) performing the electromagnetic transient computations using the equivalent or the detailed network representation were developed in Matlab [18].

Case I: For Case I, the external subsystem consists of Lines 2-5 and Lines 6-13 are disconnected. It is excited at Bus II by a set of unit step functions (with different amplitudes for each phase) and the resulting voltages and currents (1000 data points each) are used to calculate the equivalent for each mode. To have at least 15 time steps representing the travel time of the shortest transmission line, a $20-\mu$ s time step is used. As a measure of accuracy, the overall fitting error $F_{\text {err }}$ is calculated (using voltages as an example) as

$$
F_{\text {err }}=\left\|V_{e q}-V_{f n}\right\| /\left\|V_{f n}\right\|
$$

where the subscripts $e q$ and $f n$ refer to the sequences calculated using the equivalent and the full network representation, respectively. The errors for the admittance values at zero and power frequencies $Y_{\omega}$ in (14) are presented as percent deviations. The criterion for computational efficiency is the number of floating point operations (flops) needed to perform a transient calculation. Instead of time comparisons, flops are chosen because they do not depend on the platform or the compiler used.

Two equivalents, nonsparse and sparse, are calculated. The first step is to determine $p$. For the line mode, the initial value of $p$, obtained from the SVD of $C_{L}$ (built for $p=400$ ), is 167 . Based on the two limits of the network "length," it should be between 150 and 230 . Using $p=167$ as a starting point, the lower value of $p$ is determined to be 160 . This value provides a fitting error less than $10^{-5}$ and the corresponding equivalent is stable and passive. To obtain a sparse equivalent $N_{S}$ (number of nonzero coefficients) is initially set to five and the iterative procedure is interrupted when: 1 ) the fitting error is lower than 0.01 and 2) both stability and passivity criteria are satisfied. These conditions are fulfilled at $p=160$ and $N_{S}=17$. The initial order for the ground mode is determined based on the value of $p$ for the line mode and the difference in the travel times of the modes. The lower values determined for $p$ to have a fitting error lower than $10^{-5}$ and a passive and stable equivalent are 235 for the nonsparse and 270 with $N_{S}=43$ for the sparse equivalent. 
TABLE I

EQUIVALENT CHARACTERISTICS-CASE I, LINE MODE

\begin{tabular}{c|c|c|c|c}
\hline \multicolumn{5}{c}{ Unconstrained Solution of $(4)$} \\
\hline & $F_{\text {err }}$ & $0 \mathrm{~Hz}$ & \multicolumn{2}{|c}{$60 \mathrm{~Hz}$ error (\%) } \\
\cline { 4 - 5 } Equivalent: & (current) & error (\%) & $\operatorname{Re}\{Y(j \omega)\}$ & $\operatorname{Im}\{Y(j \omega)\}$ \\
\hline Non-sparse & $2.75 \mathrm{e}-6$ & 0.0499 & -0.0052 & -0.0004 \\
Sparse. & 0.00328 & -0.4977 & 1.5826 & -0.0617 \\
\hline \multicolumn{5}{|c}{ Constrained Solution of $(4)$} \\
\hline Sparse & 0.00307 & -0.0010 & -0.0010 & -0.0010 \\
\hline
\end{tabular}

TABLE II

EQuivalent ChaRACTERISTICS-CASE I, GROUND MODE

\begin{tabular}{c|c|c|c|c}
\hline \multicolumn{5}{c}{ Unconstrained Solution of (4) } \\
\hline & $F_{\text {er }}$ & $0 \mathrm{~Hz}$ & \multicolumn{2}{c}{$60 \mathrm{~Hz}$ error $(\%)$} \\
\cline { 4 - 5 } Equivalent: & (current) & error $(\%)$ & $\operatorname{Re}\{Y(j \omega)\}$ & $\operatorname{Im}\{Y(j \omega)\}$ \\
\hline Non-sparse & $3.12 \mathrm{e}-6$ & 0.9353 & 0.2562 & 0.2851 \\
Sparse. & 0.00729 & -0.5215 & 0.9092 & -0.6811 \\
\hline \multicolumn{5}{c}{ Constrained Solution of $(4)$} \\
\hline Sparse & 0.00974 & -0.0500 & 0.1471 & -0.0272 \\
\hline
\end{tabular}

TABLE III

Overall FitTing ERRor $\left(F_{\text {crr }}\right)$, CASE I

\begin{tabular}{c|c|c|c}
\hline & Phase $\mathbf{A}, V_{\text {send }}$ & Phase $\mathbf{B}, V_{\text {send }}$ & Phase $\mathbf{C}, V_{\text {send }}$ \\
\hline Non-Sparse & $1.2261 \mathrm{e}-5$ & $6.3275 \mathrm{e}-6$ & $2.4637 \mathrm{e}-5$ \\
Sparse & $1.6619 \mathrm{e}-4$ & $1.2841 \mathrm{e}-4$ & $2.1738 \mathrm{e}-4$ \\
\hline & Phase A, $V_{\text {rec. }}$ & Phase $\mathbf{B}, V_{\text {rec }}$ & Phase $\mathbf{C}, V_{\text {rec }}$ \\
\hline Non-Sparse & $4.3653 \mathrm{e}-5$ & $2.2506 \mathrm{e}-5$ & $8.7535 \mathrm{e}-5$ \\
Sparse & $5.1895 \mathrm{e}-4$ & $3.7427 \mathrm{e}-4$ & $8.1499 \mathrm{e}-4$ \\
\hline & Phase A, $I_{\text {send. }}$ & Phase $\mathbf{B}, I_{\text {send }}$ & Phase $\mathbf{C}, I_{\text {send }}$ \\
\hline Non-Sparse & $1.1177 \mathrm{e}-4$ & $6.7304 \mathrm{e}-5$ & $2.0426 \mathrm{e}-4$ \\
Sparse & 0.0015 & 0.0014 & 0.0018 \\
\hline
\end{tabular}

The tolerances for the admittance values at 0 and $60 \mathrm{~Hz}$ in the calculation of the sparse equivalent, are set to $0.001 \%$ and $0.2 \%$ for the line and ground modes, respectively.

Tables I and II present the characteristics of the equivalents for the line and ground modes corresponding to $20 \mathrm{~ms}$ of data. These tables provide: 1) fitting error and 2) admittance error at zero and power frequencies. Tables I and II also compare constrained and unconstrained solutions of (4). From these tables, one concludes that: 1) high accuracy for the admittance values of the sparse equivalent at zero and power frequencies can be obtained at low fitting error and 2) despite a very good fitting, the errors for the admittance values of the nonsparse equivalent at 0 and $60 \mathrm{~Hz}$ are noticeable, particularly for the ground mode.

Table III shows the overall fitting error $F_{\text {err }}$, based on comparisons of the detailed network transient response with that of the study subsystem plus equivalent of the external subsystem. Both nonsparse and sparse equivalents are used. Sending and receiving buses correspond to Buses I and II of Fig. 2, respectively. The transient response is due to the energization of the network at Bus I by three unit step voltages corresponding to Phases A, $\mathrm{B}$, and C. $F_{\text {err }}$ is calculated for $20 \mathrm{~ms}$ with the simulation step of $20 \mu$ s. Table III shows that both equivalents provide acceptable results in terms of accuracy.

Regarding the computational efficiency, a comparison of the flops needed for the simulation of the energization process is
TABLE IV

FLOP COMPARISONS-FD MODEL, CASE I

\begin{tabular}{c|c|c}
\hline & Mflops(absolute) & Mflops(relative) \\
\hline Full Network & 12.5574 & 1.0000 \\
\hline $\begin{array}{c}\text { (Line 1)+(Non- } \\
\text { Sparse Equiv.) }\end{array}$ & 10.5017 & 0.8363 \\
\hline $\begin{array}{c}\text { (Line 1) + (Sparse } \\
\text { Equiv.) }\end{array}$ & 3.9777 & 0.3168 \\
\hline
\end{tabular}

TABLE V

FLOP COMPARISONS-CP MODEL, CASE I

\begin{tabular}{c|c|c}
\hline & Mflops(absolute) & Mflops(relative) \\
\hline Full Network & 10.1796 & 1.0000 \\
\hline $\begin{array}{c}\text { (Line 1)+ (Non- } \\
\text { Sparse Equiv.) }\end{array}$ & 8.1206 & 0.7977 \\
\hline $\begin{array}{c}\text { (Line 1)+(Sparse } \\
\text { Equiv.) }\end{array}$ & 1.5870 & 0.1559 \\
\hline
\end{tabular}

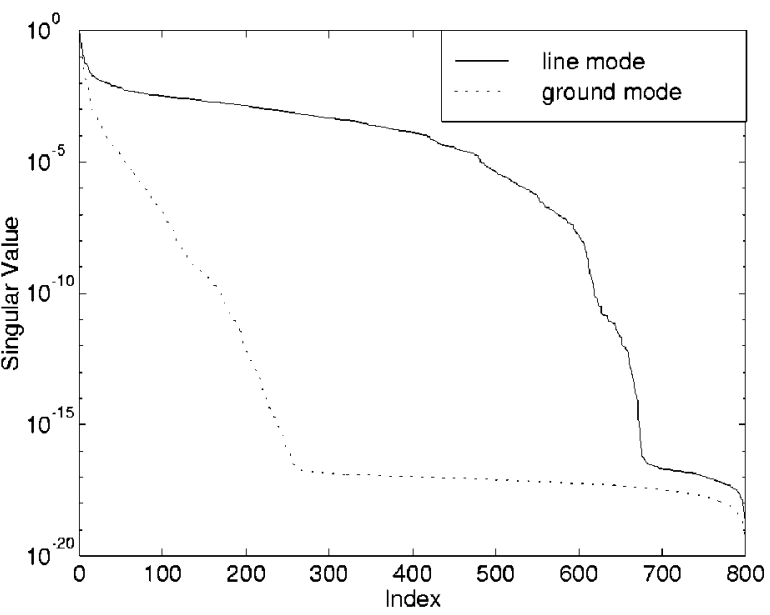

Fig. 3. SVD for the partition $C_{L}(p=800)$ for the 12 transmission-line network of Case II.

provided in Tables IV and V. The results are for the calculation of 2000 steps. For the results of Tables IV and V, FD and constant parameter (CP) models of the study subsystem line are used, respectively. These tables show that using the nonsparse equivalent has a relatively small impact on the computational efficiency. To the contrary, the sparse equivalent exhibits major effect on the efficiency.

Case II: For Case II, the study and external subsystems consist of Line 1 and Lines 2-13, respectively. To determine $p$ for the line mode, an SVD analysis of $C_{L}$ (built for $p=800$ ), Fig. 3, indicates a value of 650 and the two limits for the network "length" give a range from 310 to 680 . It should be noted that the number of significant singular values for the ground mode for Case II, when compared to Case I, is much lower than that of the line mode. This is due to the losses and the increased length of the network and results in a pronounced ill-conditioning of (4) for the ground mode. Initially, $p=650$ is chosen as the order of the equivalents for both modes. Although the equivalents for the line and ground modes are passive and result in a small fitting error, they are unstable. Several values for $p$ were examined, but a stable equivalent could not be obtained. Furthermore, a sparse equivalent was searched for values of $N_{S}$ from five to 250 . The 
TABLE VI

EQuivalent CHARACTERISTICS-CASE II, LINE AND GROUND MODES

\begin{tabular}{c|c|c|c|c}
\hline \multicolumn{5}{|c}{ Unconstrained Solution } \\
\hline NON-sparse & $F_{\text {err }}$ & $0 \mathrm{~Hz}$ & \multicolumn{2}{|c}{$60 \mathrm{~Hz}$ error $(\%)$} \\
\cline { 4 - 5 } Equivalent & (current) & error $(\%)$ & $\operatorname{Re}\{Y(\mathrm{j} \omega)\}$ & $\operatorname{Im}\{Y(\mathrm{j} \omega)\}$ \\
\hline LINE mode & $1.09 \mathrm{e}-8$ & -0.1886 & $0.098 \mathrm{e}-3$ & $-0.106 \mathrm{e}-3$ \\
\hline GROUND mode & $1.59 \mathrm{e}-2$ & -0.6685 & 0.2302 & -0.1953 \\
\hline
\end{tabular}
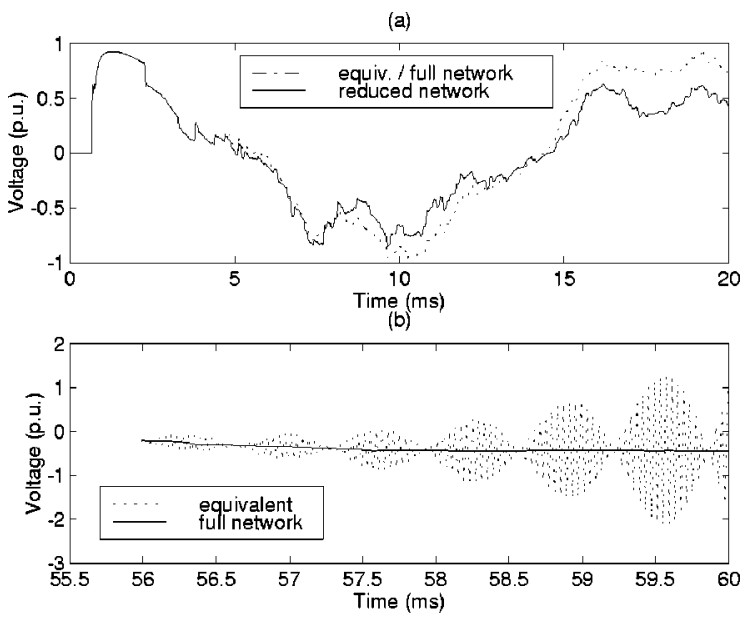

Fig. 4. Voltages at Bus II (Phase A) due to the monopolar energization of the network for Case II.

search was unsuccessful since none of the conditions, i.e., fitting error, stability, and passivity could be met. This is a limitation of the presented methodology and an alternative one will soon be reported for the case of large networks.

Table VI shows the characteristics of the nonsparse equivalents for the line and ground modes. The energization transients (similar to that of Case I) for the full network and study subsystem plus nonsparse equivalent are also computed. The flops requirement of the latter case, normalized with respect to the former, is 0.7606 .

Finally, the transient calculations due to a monopolar switching operation performed at Bus I of the network of Fig. 2 are presented for the: 1) full network; 2) reduced network; and 3) equivalent plus study subsystem. FD models are used for the transmission lines. The reduced network is obtained replacing Lines $8-13$ by the characteristic impedance of Line 8. The energization is done on Phase A, while Phase B is left open and Phase $\mathrm{C}$ is short circuited. The voltages at Bus II (Phase A) are shown in Fig. 4, where the curves for conditions 1 and 3 above are on top of each other. Comparing the curves shown in Fig. 4(a), one concludes that it is important to model the full network, as the transient voltages can be noticeably different. The effect of the equivalent's instability is shown in Fig. 4(b). The voltage curve corresponding to the equivalent starts to increase in amplitude around $56 \mathrm{~ms}$ while that for the full network tends to the steady state value of the power frequency. This also justifies the need for a procedure capable of producing an efficient, stable, and passive equivalent for large networks.

\section{CONCLUSION}

The paper presents a methodology for calculating sparse network equivalents based on time-domain fitting. A constrained least squares approach for the fitting process is used. This approach enforces the accuracy of the admittance values at zero and power frequencies. Criteria for checking stability and passivity of the equivalent are also formulated.

The results show the superior computational efficiency of the sparse equivalent for transient calculations when compared to either the nonsparse equivalent or the full network representation. The study results indicate that the overall fitting error of the sparse equivalent is satisfactory.

The presented methodology could not be successfully applied to deduce equivalents for large networks. The solution to this problem will be presented in a sequel to this paper.

\section{ACKNOWLEDGMENT}

W. C. Boaventura would like to thank the Federal University of Minas Gerais for the study leave from the University of Toronto.

\section{REFERENCES}

[1] A. S. Morched and V. Brandwajn, "Transmission network equivalents for electromagnetic transient studies," IEEE Trans. Power App. Syst., vol. PAS-102, pp. 2984-2994, Sept. 1983.

[2] A. S. Morched, J. H. Ottevangers, and L. Marti, "Multi-port frequency dependent network equivalents for the EMTP," IEEE Trans. Power Delivery, vol. 8, pp. 1402-1412, July 1993.

[3] J.-H. Hong and J.-K. Park, "A time-domain approach to transmission network equivalents via Prony analysis for electromagnetic transients analysis," IEEE Trans. Power Syst., vol. 10, pp. 1789-1797, Nov. 1995.

[4] T. Noda, N. Nagaoka, and A. Ametani, "Phase domain modeling of frequency-dependent transmission lines be means of an ARMA model," IEEE Trans. Power Delivery, vol. 11, pp. 401-411, Jan. 1996.

[5] H. Okamoto, A. Kurita, J. J. Sanchez-Gasca, K. Clark, N. W. Miller, and J. H. Chow, "Identification of equivalent linear power system models from electromagnetic transient time domain simulations using Prony's method," in Proc. 35th Conf. Decision and Control, Kobe, Japan, Dec. 1996, pp. 3857-3863.

[6] - "Identification of low order linear power system models from EMTP simulations using Steiglitz-McBride algorithm," IEEE Trans. Power Syst., vol. 13, pp. 422-427, May 1998.

[7] J. R. Smith, J. F. Hauer, and D. J. Trudnowski, "Transfer function identification in power system applications," IEEE Trans. Power Syst., vol. 8, pp. 1282-1290, May 1993.

[8] J. J. Sanchez-Gasca and J. H. Chow, "Performance comparison of three identification methods for the analysis of electromechanical oscillations," IEEE Trans. Power Syst., vol. 14, pp. 995-1002, Aug. 1999.

[9] J. R. Marti, "Accurate modeling of frequency-dependent transmission lines in electromagnetic transient simulations," IEEE Trans. Power App. Syst., vol. PAS-101, pp. 147-155, Jan. 1982.

[10] J. Lin and J. R. Marti, "Implementation of the CDA procedure in the EMTP," IEEE Trans. Power Syst., vol. 5, pp. 394-402, May 1990.

[11] A. Semlyen and F. de Leon, "Computational of electromagnetic transients using dual or multiple time steps," IEEE Trans. Power Syst., vol. 8, pp. 1274-1281, Aug. 1992.

[12] G. Angelidis and A. Semlyen, "Direct phase-domain calculation of transmission line transients using two-sided recursions," IEEE Trans. Power Delivery, vol. 10, pp. 941-949, Apr. 1995.

[13] T. Henriksen, "Including high order rational functions in EMTP a comparison between alternative methods with emphasis on accuracy," IEEE Trans. Power Delivery, vol. 12, pp. 372-379, Jan. 1997.

[14] T. Noda, N. Nagaoka, and A. Ametani, "Further improvements to a phase-domain ARMA line model in terms of convolution, steady-state initialization, and stability," IEEE Trans. Power Delivery, vol. 12, pp. 1327-1334, July 1997. 
[15] H. V. Nguyen, H. W. Dommel, and J. R. Marti, "Direct phase-domain modeling of frequency-dependent overhead transmission lines," IEEE Trans. Power Delivery, vol. 12, pp. 1335-1342, July 1997.

[16] A. V. Oppenheim and R. W. Schafer, Discrete-Time Signal Processing. Englewood Cliffs, NJ: Prentice-Hall, 1989.

[17] R. Prony, "Essai expérimental et analytique sur les lois de la dilatabilité des fluides élastiques et sur celles de la force expansive de la vapeur d'alcool à différentes températures" (in French), J. l'Ecole Polytech., vol. 1, no. 2, pp. 24-76, 1795.

[18] Matlab-Language Reference Manual -V. 5, The MathWorks Inc., June 1997.

[19] M. L. Van Blaricum and R. Mittra, "Problems and solutions associated with Prony's method for processing transient data," IEEE Trans. Antennas Propagat., vol. AP-16, pp. 174-182, Jan. 1978.

[20] B. Gustavsen and A. Semlyen, "Rational approximation of frequency domain responses by vector fitting," IEEE Trans. Power Delivery, vol 5, pp. 1052-1061, July 1999.

Wallace do Couto Boaventura (M'94) was born in Brazil in 1965. He received the B.Sc. and M.Sc. degrees from the Federal University of Minas Gerais, Belo Horizonte, MG, Brazil, in 1988 and 1990, respectively. He is currently working toward the Ph.D. degree at the University of Toronto, Toronto, ON Canada.

$\mathrm{He}$ is currently on leave from the Federal University of Minas Gerais, where he has been an Assistant Professor since 1992.
Adam Semlyen (SM'70-F'88-LF'97) was born in Romania in 1923. He received the Dipl.Ing. degree and the Ph.D. degree.

In 1969, he joined the University of Toronto, where he is a Professor in the Department of Electrical and Computer Engineering, emeritus since 1988. His current research interests include steady-state and dynamic analysis as well as computation of electromagnetic transients in power systems.

M. Reza Iravani (M'85-SM'00) received the B.Sc. degree in 1976, the M.A.Sc. degree in 1981, and the Ph.D. degree in 1985, all in electrical engineering.

$\mathrm{He}$ is currently a Professor with the University of Toronto, Toronto, ON Canada. His current research interests include power system transients and power electronics.

Amauri Lopes (S'75-M'80) received the B.Sc. degree in 1972, the M.Sc. degree in 1974, and the Ph.D. degree in 1982, all in electrical engineering, from the University of Campinas.

Since 1973, he has been with the Electrical and Computer Engineering School, where he is currently an Associate Professor. His current research interests include digital signal processing and wireless communications. 